\title{
Electromagnetic Properties of Inner Double Walled Carbon Nanotubes Investigated by Nuclear Magnetic Resonance
}

\author{
M. Bouhrara, ${ }^{1}$ E. Abou-Hamad, ${ }^{2,3}$ G. Alabedi, ${ }^{1}$ I. Al-Taie, ${ }^{1}$ \\ Y. Kim, ${ }^{4}$ T. Wågberg, ${ }^{5}$ and C. Goze-Bac ${ }^{2}$ \\ ${ }^{1}$ Design Nano Applications Division (DNA), Research \& Development Center, Saudi Aramco, Dhahran 31311, Saudi Arabia \\ ${ }^{2}$ nanoNMRI group, UMR5587, University of Montpellier II, 34095 Montpellier, France \\ ${ }^{3}$ KAUST Catalysis Center (KCC), King Abdullah University of Science and Technology, Thuwal 23955-6900, Saudi Arabia \\ ${ }^{4}$ Department of Materials Science and Engineering, University of Pennsylvania, Philadelphia, PA 19104, USA \\ ${ }^{5}$ Department of Physics, Umeå University, 90187 Umeå, Sweden
}

Correspondence should be addressed to E. Abou-Hamad; edy.abouhamad@kaust.edu.sa

and C. Goze-Bac; goze@univ-montp2.fr

Received 18 July 2013; Accepted 30 September 2013

Academic Editor: Tianxi Liu

Copyright (C) 2013 M. Bouhrara et al. This is an open access article distributed under the Creative Commons Attribution License, which permits unrestricted use, distribution, and reproduction in any medium, provided the original work is properly cited.

\begin{abstract}
The nuclear magnetic resonance (NMR) analytical technique was used to investigate the double walled carbon nanotubes (DWNTs) electromagnetic properties of inner walls. The local magnetic and electronic properties of inner nanotubes in DWNTs were analyzed using $25 \%{ }^{13} \mathrm{C}$ enriched $\mathrm{C}_{60}$ by which the effect of dipolar coupling could be minimized. The diamagnetic shielding was determined due to the ring currents on outer nanotubes in DWNTs. The NMR chemical shift anisotropy (CSA) spectra and spin-lattice relaxation studies reveal the metallic properties of the inner nanotubes with a signature of the spin-gap opening below $70 \mathrm{~K}$.
\end{abstract}

\section{Introduction}

With their special properties, such as superconductivity or magnetic shielding effect, extensive studies of double walled carbon nanotubes(DWNTs) have been conducted theoretically as well as experimentally [1-8]. Nuclear magnetic resonance (NMR) study, which is one of the most important tools to investigate their electronic and local magnetic properties, however, has been limited due to transition metal impurities used as catalyst particles during synthesis. Recently, Singer et al. used DWNTs made from $89 \%$ enriched $\mathrm{C}_{60}$ peapods by high temperature annealing, so that encapsulated fullerenes dominate NMR signal [9]. From ${ }^{13} \mathrm{C}$ relaxation study, they found that all the inner nanotubes show 1D metallic Luttinger-liquid behavior, followed by the spin-gap opening at low temperature. Besides the interesting $1 \mathrm{D}$ behavior, there is still much more information in DWNTs to be explored. For example, a theoretical study by Marques et al. predicts that inner nanotubes would experience diamagnetic shifts due to ring currents from $\pi$ electrons on outer nanotubes [10].
In our study, with the novel magnetic filtration purification method, we could obtain ferromagnetic catalyst-free diamagnetic single walled carbon nanotubes (SWNTs). They produce well resolved NMR lines despite natural carbon abundance [11, 12]. This enables us to investigate the local magnetic and electronic properties of inner nanotubes in DWNTs using $25 \%{ }^{13} \mathrm{C}$ enriched $\mathrm{C}_{60}$ by which the effect of dipolar coupling could be minimized. As predicted by Marques et al., we found the diamagnetic shielding from outer nanotubes in DWNTs [10]. The CSA NMR spectra and spin-lattice relaxation studies on DWNTs revealed the metallic properties of the inner nanotubes with a signature of the spin-gap opening below $70 \mathrm{~K}$ as claimed by Singer et al. [9].

\section{Experimental Procedures}

The starting material, SWNTs and $25 \%{ }^{13} \mathrm{C}_{\text {enriched }} \mathrm{C}_{60}$, was purchased from Carbon Solution Inc. and MER Corporation, 
respectively. The magnetic purification is well described in [12]. Briefly, SWNTs were oxidized in air for 10 minutes at $600^{\circ} \mathrm{C}$ following bath sonication in hydrochloric acid for 40 minutes at $650^{\circ} \mathrm{C}$ to remove bare $\mathrm{Ni} / \mathrm{Y}$ catalyst particles. After the acid treated materials were dispersed in dimethylformamide (DMF) by bath sonication, the solution was filtered through the magnetic field of 1.1 Tesla. Peapods were then prepared by vapor phase filling for $10 \mathrm{hrs}$ at $650^{\circ} \mathrm{C}$, following $1 \mathrm{hr}$ after annealing in dynamic vacuum to remove excessive enriched fullerenes. Finally, DWNTs were fabricated by annealing the peapods dynamic vacuum for $48 \mathrm{hrs}$ at $1250^{\circ} \mathrm{C}$. With Renishaw 100 spectrometer operating with red laser $(633 \mathrm{~nm})$ at room temperature, the development of inner tubes in DWNTs was verified. For the representative images of peapods and DWNTs, JOEL 2010 transmission electron microscope was used. ${ }^{13} \mathrm{C}$ NMR experiments were carried out in nanoNMRI group, Montpellier, using a Bruker ASX200 spectrometer at magnetic field of $4.2 \mathrm{~T}$ and Larmor frequency of $50.3 \mathrm{MHz}$. The ${ }^{13} \mathrm{C}$ NMR spectrum was performed using Han echo synchronized with a rotor spinning at $10 \mathrm{KHz}$. The spin-lattice relaxation time was measured by saturation-recovery technique. Temperature studies in NMR were performed in a sealed glass tube after being evacuated overnight at a dynamic vacuum of $10^{-8}$ Torr. ${ }^{13} \mathrm{C}$ MAT NMR experiment was carried out using the 5-pulse sequence. Data acquisition was performed directly after the last $\pi / 2$ pulse. The MAT rotor frequency was $150 \mathrm{~Hz}$ and $\tau$ varied from $0.1 \mathrm{~s}$ to $6 \mathrm{~s}$ within 40 steps. The free induction decay (FID) acquisition during the acquisition included 1024 points with a dwell time of $4 \mu \mathrm{s}$. A two-dimensional Fourier transform technique represents the isotropic chemical shift projection in one frequency dimension (F1) and the static anisotropic powder pattern along the other frequency axis (F2) [13-15].

\section{Results and Discussion}

In Figure 1, we show the Raman spectra of SWNTs (I), peapod (II), and DWNTs treated by annealing peapod samples (III). The radial breathing mode positions of the SWNTs at 150 and $167 \mathrm{~cm}^{-1}$ indicate the presence of tubes with diameters of 1.57 and $1.46 \mathrm{~nm}$, respectively [16]. This is in good agreement with the estimated tube diameter from the HR-TEM. In addition to the SWNT Raman modes, we see for the untreated peapod sample weak signals from the $\mathrm{C}_{60}$ molecules confined in the SWNTs. The positions of the $A_{g}(1)$ and the $A_{g}(2)$ modes at 484 and $1450 \mathrm{~cm}^{-1}$ are downshifted compared to pristine $\mathrm{C}_{60}$ due to the ${ }^{13} \mathrm{C}$ enrichment of the $\mathrm{C}_{60}$ molecules. The DWNTs samples show additional Raman features in the spectral region of $250-350 \mathrm{~cm}^{-1}$. They originate from the inner nanotube after annealing fullerene, whose average diameter is $0.75 \mathrm{~nm}$. This compares with the average diameter of inner tubes from the direct measurement in TEM images. Since Raman spectroscopy provided only limited information regarding composition of the sample, the most detailed information was obtained by NMR spectroscopy.

The NMR study was performed using Han echo synchronized with a rotor spinning at $10 \mathrm{KHz}$, on peapod, and on DWNTs synthesized from these peapods. It is possible to

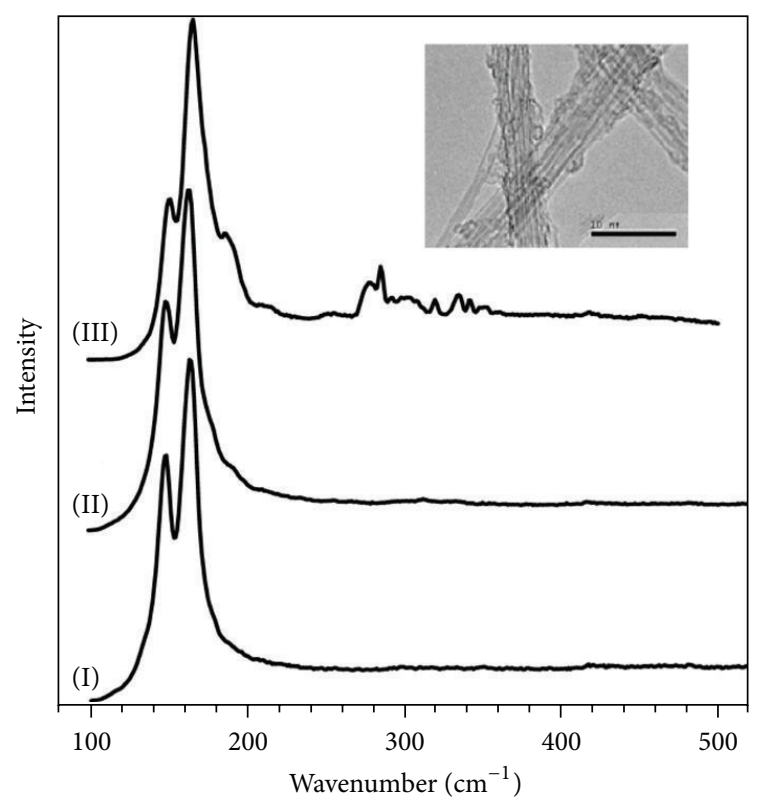

FIGURE 1: Raman spectra excited with an argon ion laser $(633 \mathrm{~nm})$ of (I) magnetically purified SWNTs, (II) peapods, and (III) DWNTs obtained by annealing peapods at $1250^{\circ} \mathrm{C}$ for 24 hours. Inset shows the TEM image of DWNTs.

assign the observed effects confidently only to molecules of $\mathrm{C}_{60}$ and nanotubes situated in the interior space of SWNT. Figure 2 shows the magic angle spinning NMR spectra of peapods (I) and DWNTs (II). The signal from the peapods can be fitted with three lines, the first at $111.3 \mathrm{ppm}(\beta$ position), a second at $118.8 \mathrm{ppm}$, and a third at $148.2 \mathrm{ppm}(\alpha-$ position). The line at $111.3 \mathrm{ppm}$ is assigned to $\mathrm{C}_{60}$ molecules in the interior of the SWNTs. This signal is diamagnetically shifted from the normal position of FCC $\mathrm{C}_{60}$, located at $143.6 \mathrm{ppm}$. The diamagnetic shift occurs due to a local magnetic field on the $\mathrm{C}_{60}$ molecules produced by ring currents on the honeycomb surface of the nanotube [10, 17-19]. The same effect has been observed for ${ }^{3} \mathrm{He}$ encapsulated inside fullerene molecules [20]. The sharp character of this line indicates that the $\mathrm{C}_{60}$ molecules are still rotating inside the carbon nanotubes ( $\beta$-position). This result is in agreement with semiempirical calculations [21] and recent experiments, $[17-19,22]$ which show that $C_{60}$ molecules inside nanotubes rotate at the NMR time scale at room temperature. The signal at $118.8 \mathrm{ppm}$ represents the NMR response of the carbons in the SWNTs $[23,24]$. Finally, the line at $148.2 \mathrm{ppm}$ represents $\mathrm{C}_{60}$ molecules at the interior of SWNTs but located at tube defects, giving rise to a slight paramagnetic shift of the NMR line position compared to pristine $\mathrm{C}_{60}$ [25]. Its significant broadening compared to freely rotating $\mathrm{C}_{60}$ suggests that the $\mathrm{C}_{60}$ molecules are no longer rotating when located in the vicinity of nanotube defects ( $\alpha$-position). The broadening indicates also that $\mathrm{C}_{60}$ molecules are not located outside the SWNT, which would show up as a narrow line at $143.6 \mathrm{ppm}$ [26]. The DWNTs result in drastic modifications of the NMR spectra. The ${ }^{13} \mathrm{C}$ NMR MAS spectrum (Figure 2, (II)) shows that the signals of $\alpha$ and $\beta$ molecules keep 


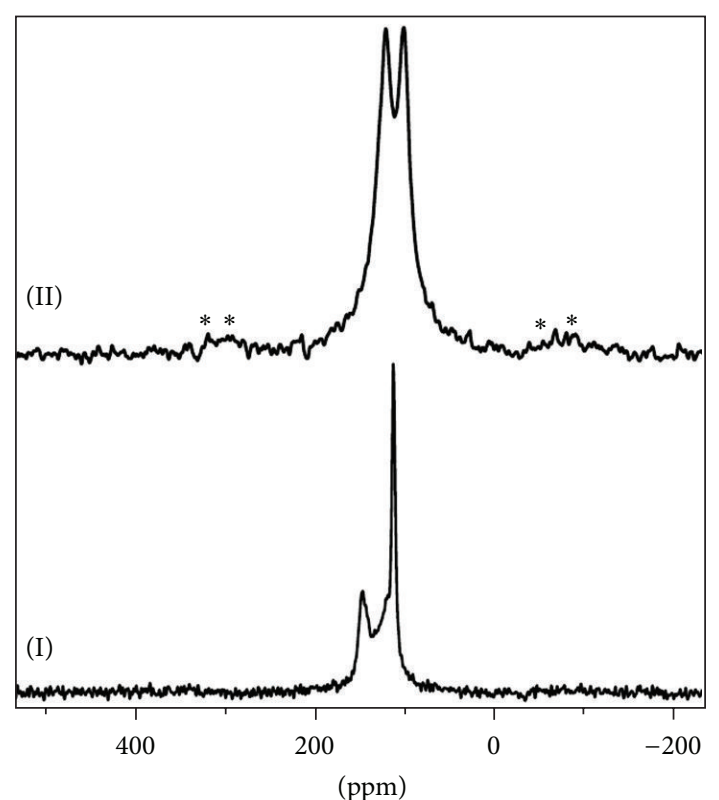

FIGURE 2: High resolution ${ }^{13} \mathrm{C}$ MAS NMR spectra of (I) peapods and (II) DWNTs. The stars symbols are for the spinning sidebands. Spinning rate is about $10 \mathrm{KHz}$.

their individual ratio but the line positions and their fwhm are changed. Mention that the $\alpha$ and $\beta$ shifted fullerenes transformed into shifted inner nanotubes. Fitting the NMR data for the DWNTs (Figure 3) can be done using one Lorentzian line at $118.8 \mathrm{ppm}$ representing the outer SWNTs and a distribution of Lorentzian lines centered around 125.8 and $100 \mathrm{ppm}$ representing the inner nanotubes at $\alpha$ and $\beta$ positions, respectively [17-19]. This fit allows us to estimate that the SWNTs provide a $16 \pm 2 \%$ contribution to the measured signal, whereas the remaining $84 \%$ comes from inner nanotubes. This result agrees well with a 70\% filling factor of peapods and a $25 \%{ }^{13} \mathrm{C}$ enrichment of encapsulated $\mathrm{C}_{60}$ [12]. As predicted by Marques et al. [10], the peak at $100 \mathrm{ppm}$ is assigned to diamagnetically shifted inner nanotubes ( $\beta$-position) due to ring current from $\pi$ electrons on outer nanotubes. The other peak at $125.8 \mathrm{ppm}$ can be deconvoluted into two contributions, one from paramagnetically shifted inner nanotube ( $\alpha$-position) close to defects on outer nanotubes and the other from outer nanotubes themselves. However, the diamagnetic shift in DWNTs $(-26.6 \mathrm{ppm})$ is smaller than that in peapods $(-36.9 \mathrm{ppm})$. In the current study, the magnitude of a diamagnetic shift is measured by the gap between paramagnetically and diamagnetically shifted peaks. The former shift is sensitive to the content of defects on outer nanotubes. The lesser number of defects in DWNTs than those in peapods will lead to the smaller paramagnetic shift and hence smaller gap. This is due to the heat treatment during the DWNTs production.

The difference between the inner nanotubes (125.8 ppm), relative to the outer nanotubes (118.8 ppm), is expected due to the paramagnetic shift for carbon located at tube defects and the smaller diameter in the former which modify the electronic structure of $\pi$ orbital [27-32].

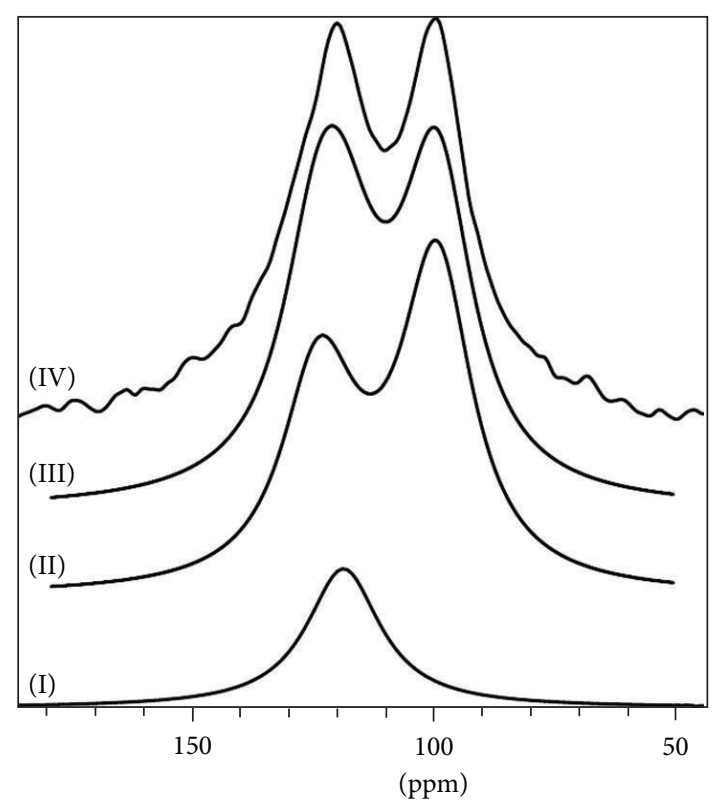

FIgURE 3: Fit of high resolution ${ }^{13} \mathrm{C}$ MAS NMR spectrum of DWNTs. Outer SWNTs (I), paramagnetically and diamagnetically shifted inner nanotubes (II), fitted curve (III), and experimental NMR spectrum (IV).

For the investigation of shift anisotropy the magic angle turning (MAT) technique was applied. Two-dimensional Fourier transformation as a function of $\tau$ and $t_{a}$ results in the 2D-MAT spectrum of DWNTs in Figure 4. It is important to note that projections of the $2 \mathrm{D}$ spectrum onto the two axes of the contour plot do not directly represent the isotropic and chemical shift anisotropy (CSA) patterns, since data acquisition is already started after the last $\pi / 2$-pulse of the MAT pulse sequence. Therefore, the scaling of the axis in Figure 4(a) is displayed in arbitrary units. Projecting the spectrum onto the acquisition dimension axis at an angle $\arctan \left(\zeta_{2} / 3 \zeta_{1}\right)$ will result in the isotropic-shift spectrum, while projection on a perpendicular axis will result in the CSA powder pattern. $\zeta_{1 / 2}$ is defined by the number of acquisition points and dwell times given by $\tau$ and $t_{a}$ [14]. In the 2D spectrum two main peaks (A) and (B) are clearly visible, indicating the $\alpha$ and $\beta$ positions of the inner nanotubes. Projecting the spectrum onto the acquisition dimension axis gives the same isotropic ${ }^{13} \mathrm{C}$ NMR spectrum shown in Figure 2 (II). Projecting the spectrum on a perpendicular axis yields the anisotropic (CSA) ${ }^{13} \mathrm{C}$ NMR spectrum in Figure 4(b) of DWNTs. However, the CSA NMR spectra exhibit a clear anisotropy reduction compared to SWNTs due to change into metallic-like shift anisotropy as reported by Goze-Bac et al. and Latil et al. [23, 24], which supports the results of Singer et al. that claimed that all inner nanotubes are metallic [9]. There are two known contributions to the total ${ }^{13} \mathrm{C}$ NMR shift of metallic tubes, namely, the Knight shift $K$ and the chemical shift $\sigma$, where the Knight shift arises from the hyperfine coupling of the nuclei to conduction electron spins and the chemical shift summarizes all contributions from orbital currents due to local magnetic 


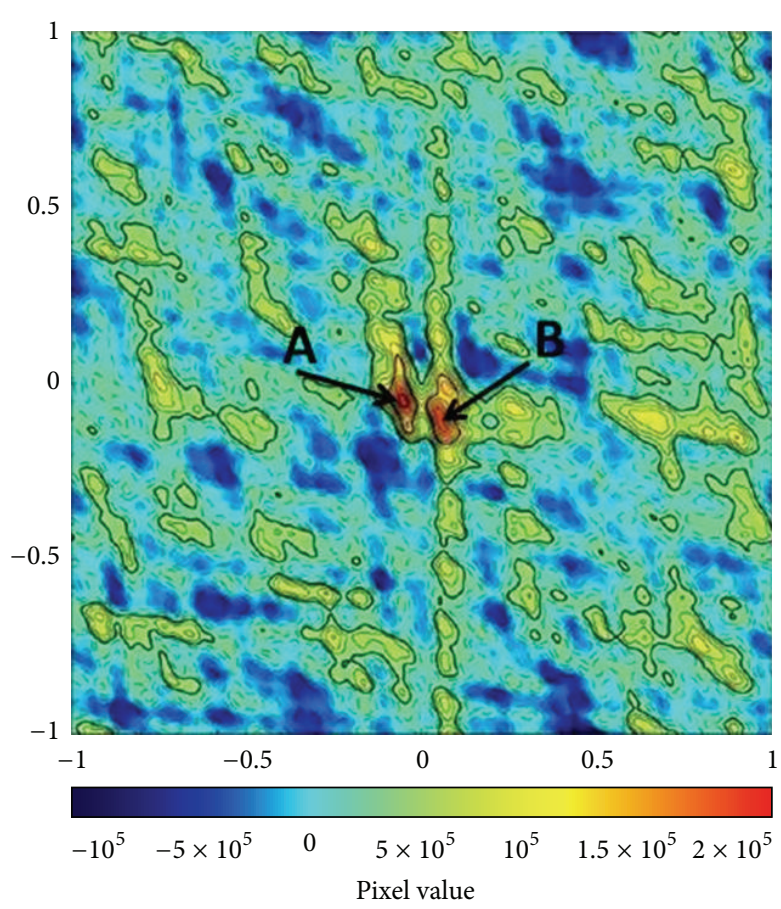

(a)

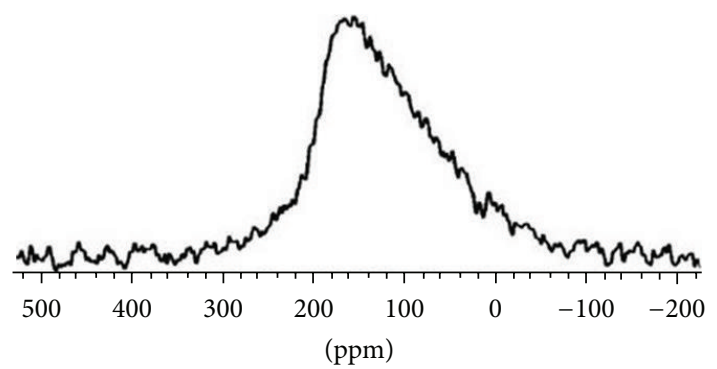

(b)

Figure 4: (a) Room temperature ${ }^{13} \mathrm{C}$ MAT NMR spectrum of DWNTs, (A) and (B) can be assigned to the $\alpha$ and $\beta$ positions of the inner nanotubes. (b) Anisotropic 13C NMR spectrum. The spectrum was extracted from the corresponding 2D-MAT experiment.

fields. Both Knight shift $K$ and spin-lattice relaxation time $T_{1}$ relaxation are affected by the density of states (DOS) at the Fermi surface $n\left(E_{F}\right)$ shift and relaxation are dominated by the magnetic interaction with the conduction electrons of the metal [33]. In order to go further in our electronic study and to obtain a better estimate of $n\left(E_{F}\right)$, spin-lattice relaxation time measurements on DWNTs were applied. At room temperature, in the inset of Figure 5, the observed relaxation curves signal a two-component model with one slow relaxing and one fast relaxing component, which can be rationalized by different types of inner nanotubes. Recent NMR studies on pristine SWNTs [34] and DWNTs [35] have used partly different models based on a stretched distribution of $T_{1}$ values around one average. Indeed, the shape of MAS NMR spectrum with delay time of $200 \mathrm{~ms}$ shows the clear difference compared to that with $20 \mathrm{~s}$ delay as shown in Figure 5, which forced us to use to fit the magnetization recovery, $M(t)$, with double exponential function of the type:

$$
M=M_{s}\left(1-e^{-t / T_{1 s}}\right)+M_{m}\left(1-e^{-t / T_{1 m}}\right),
$$

where $M_{s}$ and $M_{m}$ are the equilibrium magnetizations for the two components. The relative magnitudes, $M_{s} / M_{0}$ and $M_{m} / M_{0}$, with $M_{0}=M_{S}+M_{m}$ do not differ in a systematic way and are determined to be $75 \% \pm 7 \%$ and $25 \% \pm 7 \%$. The slow and fast relaxing components derived from the fit at room temperature result in $T_{1 \mathrm{~s}}=5 \mathrm{~s}$ and $T_{1 \mathrm{~m}}=0.2 \mathrm{~s}$. The two components are very small compared to SWNTs $[23,24]$ and are in good agreement with metallic doping nanotube at high intercalations level $[36,37]$ due to high density of states (DOS) of the metallic tubes.

${ }^{13} \mathrm{C} \mathrm{NMR} T_{1}$ measurements as a function of temperature in Figure 6 can be expressed as

$$
\frac{1}{T T_{1}}=\frac{2 \pi k_{B}}{\hbar} A_{\text {iso }}^{2} n\left(E_{F}\right)^{2},
$$

where $A_{\text {iso }}$ is the isotropic hyperfine coupling constant [38]. We assume a hyperfine coupling of $A_{\text {iso }}=8.2 \times 10^{-7}$ $\mathrm{eV}$ and fit the linear regime part of the ${ }^{13} \mathrm{C}$ NMR spinlattice relaxation. Hence, we were able to estimate the $n\left(E_{F}\right)$ of DWNTs. For the slow component we observed a linear regime following Korringa behavior, which is typical for a metallic Fermi liquid system. For the fast component we observed a Korringa behavior that decreases linearly down to $70 \mathrm{~K}$, the deviation below this temperature is the signature of the spin-gap opening as Singer et al. claimed [9]. From (2) $n\left(E_{F}\right)$ can be extracted to be 0.057 and 0.011 states $/(\mathrm{eV} \cdot \mathrm{spin} \cdot$ atom $)$, for the fast and slow relaxation respectively. Since $n\left(E_{F}\right)$ is inversely proportional to the diameter of nanotubes, theoretical $n\left(E_{F}\right)$ for $0.7 \mathrm{~nm}$ nanotubes is predicted to be 0.03 states $/(\mathrm{eV} \cdot \mathrm{spin} \cdot$ atom $)$ or higher $[7,39]$. The enhancement of the extracted $n\left(E_{F}\right)$ compared to the theoretical value is expected from the interaction with outer nanotubes or from small doping caused by charge transfer from outer to inner nanotubes [3-6]. Indeed, also Hashimoto et al. performed HR-TEM study where only the inner tube was found to have metallic geometry in random observation [3]. 


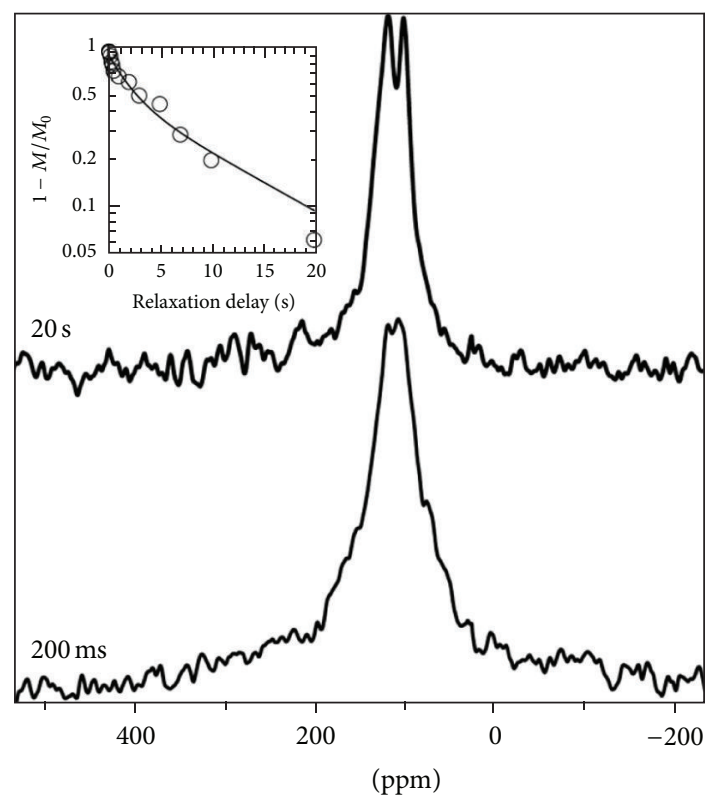

FIgURE 5: ${ }^{13} \mathrm{C}$ MAS NMR spectrum of DWNTs at two different delays. Inset ${ }^{13} \mathrm{C}$ NMR magnetization recovery at room temperature.

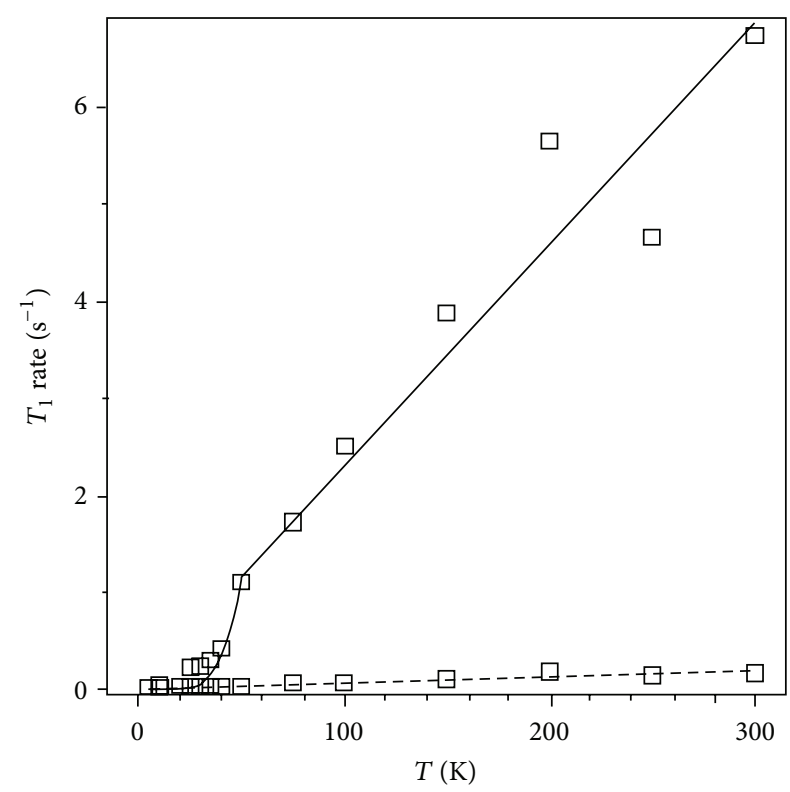

FIgURE 6: Temperature-dependent ${ }^{13} \mathrm{C}$ NMR spin-lattice relaxation rates plotted as $1 / T_{1}$ against temperature $T$ for DWNTs.

\section{Conclusion}

Using $25 \%{ }^{13} \mathrm{C}$ enriched $\mathrm{C}_{60}$ by which the effect of dipolar coupling could be minimized enables us to investigate the local magnetic and electronic properties of inner nanotubes in DWNTs. ${ }^{13} \mathrm{C}$ NMR on DWNTs produced two well resolved isotropic shifts. The one at $100 \mathrm{ppm}$ is assigned to diamagnetically shift inner nanotubes ( $\beta$-position) due to ring current from $\pi$ electrons on outer nanotubes. The other peak at $125.8 \mathrm{ppm}$ can be deconvoluted into two contributions, one from paramagnetically shifted inner nanotube $(\alpha-$ position) close to defects on outer nanotubes and the other from outer nanotubes themselves. For the investigation of shift anisotropy (CSA), the magic angle turning (MAT) technique was applied that exhibits a clear anisotropy reduction compared to SWNTs due to change into metallic-like shift anisotropy. The spin-lattice relaxation studies on DWNTs reveal a two-component model with one slow relaxing and one fast relaxing component which can be rationalized by different types of inner nanotubes. The two components are very small compared to SWNTs and are in good agreement with metallic nanotube due to high density of states (DOS) of the metallic tubes. The ${ }^{13} \mathrm{C}$ NMR $T_{1}$ measurements as a function of temperature confirm the metallic properties of the inner nanotubes with a signature of the spin-gap opening below $70 \mathrm{~K}$.

\section{Authors' Contribution}

E. Abou-Hamad and C. Goze-Bac contributed equally as corresponding authors.

\section{References}

[1] Y. Kwon and D. Tomanek, "Electronic and structural properties of multiwall carbon nanotubes," Physical Review B, vol. 58, no. 24, pp. R16001-R16004, 1998.

[2] P. H. Lambin, V. Meunier, and A. Rubio, "Electronic structure of polychiral carbon nanotubes," Physical Review B, vol. 62, no. 8, pp. 5129-5135, 2000.

[3] A. Hashimoto, K. Suenaga, K. Urita et al., "Atomic correlation between adjacent graphene layers in double-wall carbon nanotubes," Physical Review Letters, vol. 94, no. 4, Article ID 045504, 4 pages, 2005.

[4] Y. Miyamoto, S. Saito, and D. Tomanek, "Electronic interwall interactions and charge redistribution in multiwall nanotubes," Physical Review B, vol. 65, no. 4, Article ID 041402, 4 pages, 2001.

[5] S. Okada and A. Oshiyama, "Curvature-induced metallization of double-walled semiconducting zigzag carbon nanotubes," Physical Review Letters, vol. 91, no. 21, Article ID 216801, 4 pages, 2003.

[6] W. Song, M. Ni, J. Lu et al., "Electronic structures of semiconducting double-walled carbon nanotubes: important effect of interlay interaction," Chemical Physics Letters, vol. 414, no. 4-6, pp. 429-433, 2005.

[7] R. Saito, R. Matsuo, T. Kimura, G. Dresselhaus, and M. S. Dresselhaus, "Anomalous potential barrier of double-wall carbon nanotube," Chemical Physics Letters, vol. 348, no. 3-4, pp. 187193, 2001.

[8] F. Simon, C. Kramberger, R. Pfeiffer et al., "Isotope engineering of carbon anotube systems," Physical Review Letters, vol. 95, no. 1, Article ID 017401, 4 pages, 2005.

[9] P. M. Singer, P. Wzietek, H. Alloul, F. Simon, and H. Kuzmany, "NMR evidence for gapped spin excitations in metallic carbon nanotubes," Physical Review Letters, vol. 95, no. 23, Article ID 236403, 4 pages, 2005.

[10] M. A. L. Marques, M. d'Avezac, and F. Mauri, "Magnetic response and NMR spectra of carbon nanotubes from $a b$ initio 
calculations," Physical Review B, vol. 73, no. 12, Article ID 125433, 6 pages, 2006.

[11] Y. Kim and D. E. Luzzi, "Purification of pulsed laser synthesized single wall carbon nanotubes by magnetic filtration," The Journal of Physical Chemistry B, vol. 109, no. 35, pp. 1663616643, 2005.

[12] Y. Kim, O. N. Torrens, J. M. Kikkawa, E. Abou-Hamad, C. Goze-Bac, and D. E. Luzzi, "High-purity diamagnetic singlewall carbon nanotube buckypaper," Chemistry of Materials, vol. 19, no. 12, pp. 2982-2986, 2007.

[13] A. Bax, N. M. Szeverenyi, and G. E. Maciel, "Pulsed polarization transfer for ${ }^{13} \mathrm{C}$ NMR in solids," Journal of Magnetic Resonance, vol. 50, no. 2, pp. 227-232, 1982.

[14] J. Z. Hu, W. Wang, F. Liu et al., "Magic-angle-turning experiments for measuring chemical-shift-tensor principal values in powdered solids," Journal of Magnetic Resonance A, vol. 113, no. 2, pp. 210-222, 1995.

[15] Z. Gan and R. R. Ernst, "An improved 2d magic-angleturning pulse sequence for the measurement of chemical-shift anisotropy," Journal of Magnetic Resonance A, vol. 123, no. 1, pp. 140-143, 1996.

[16] S. M. Bachilo, M. S. Strano, C. Kittrell, R. H. Hauge, R. E. Smalley, and R. B. Weisman, "Structure-assigned optical spectra of single-walled carbon nanotubes," Science, vol. 298, no. 5602, pp. 2361-2366, 2002.

[17] E. Abou-Hamad, Y. Kim, A. V. Talyzin et al., "Hydrogenation of $\mathrm{C}_{60}$ in peapods: physical chemistry in nano vessels," The Journal of Physical Chemistry C, vol. 113, no. 20, pp. 8583-8587, 2009.

[18] E. Abou-Hamad, Y. Kim, T. Wågberg et al., "Molecular dynamics and phase transition in one-dimensional crystal of $\mathrm{C}_{60}$ encapsulated inside single wall carbon nanotubes," ACS Nano, vol. 3, no. 12, pp. 3878-3883, 2009.

[19] Y. Kim, E. Abou-Hamad, A. Rubio et al., "Communications: nanomagnetic shielding: high-resolution NMR in carbon allotropes," Journal of Chemical Physics, vol. 132, no. 2, Article ID 021102, 2010.

[20] T. Sternfeld, M. Saunders, R. J. Cross, and M. Rabinovitz, "The inside story of fullerene anions: a ${ }^{3} \mathrm{He}$ NMR aromaticity probe," Angewandte Chemie, vol. 42, no. 27, pp. 3136-3139, 2003.

[21] J. Chen and J. Dong, "Electronic properties of peapods: effects of fullerene rotation and different types of tube," Journal of Physics, vol. 16, no. 8, article 1401, 2004.

[22] K. Matsuda, Y. Maniwa, and H. Kataura, "Highly rotational $\mathrm{C}_{60}$ dynamics inside single-walled carbon nanotubes: NMR observations," Physical Review B, vol. 77, no. 7, Article ID 075421, 6 pages, 2008.

[23] C. Goze-Bac, S. Latil, P. Lauginie et al., "Magnetic interactions in carbon nanostructures," Carbon, vol. 40, no. 10, pp. 1825-1842, 2002.

[24] S. Latil, L. Henrard, C. Goze-Bac, P. Bernier, and A. Rubio, " ${ }^{13} \mathrm{C}$ NMR chemical shift of single-wall carbon nanotubes," Physical Review Letters, vol. 86, no. 14, pp. 3160-3163, 2001.

[25] F. López-Urías, J. A. Rodríguez-Manzo, E. Muñoz-Sandoval, M. Terrones, and H. Terrones, "Magnetic response in finite carbon graphene sheets and nanotubes," Optical Materials, vol. 29, no. 1, pp. 110-115, 2006.

[26] T. Wågberg, C. Goze-Bac, R. Röding et al., " ${ }^{13}$ C NMR on intercalated 2D-polymerised $\mathrm{C}_{60}$ and modified peapods," in Proceedings of the 17th International Winterschool/Euroconference on Electronic Properties of Novel Materials (IWEPNM '04), vol. 723 of AIP Conference Proceedings, p. 238, March 2004.
[27] E. Abou-Hamad, M.-R. Babaa, M. Bouhrara et al., "Structural properties of carbon nanotubes derived from ${ }^{13} \mathrm{C}$ NMR," Physical Review B, vol. 84, no. 16, Article ID 165417, 2011.

[28] E. Zurek, C. J. Pickard, B. Walczak, and J. Autschbach, "Density functional study of the ${ }^{13} \mathrm{C}$ NMR chemical shifts in smallto-medium-diameter infinite single-walled carbon nanotubes," The Journal of Physical Chemistry A, vol. 110, no. 43, pp. 1199512004, 2006.

[29] E. Zurek, C. J. Pickard, and J. Autschbach, "Density functional study of the ${ }^{13} \mathrm{C}$ NMR chemical shifts in single-walled carbon nanotubes with Stone-Wales defects," The Journal of Physical Chemistry C, vol. 112, no. 31, pp. 11744-11750, 2008.

[30] E. Zurek, C. J. Pickard, and J. Autschbach, "A density functional study of the ${ }^{13} \mathrm{C}$ NMR chemical shifts in fluorinated singlewalled carbon nanotubes," The Journal of Physical Chemistry A, vol. 113, no. 16, pp. 4117-4124, 2009.

[31] E. Zurek, C. J. Pickard, and J. Autschbach, "Determining the diameter of functionalized single-walled carbon nanotubes with ${ }^{13}$ C NMR: a theoretical study," The Journal of Physical Chemistry C, vol. 112, no. 25, pp. 9267-9271, 2008.

[32] D. Sebastiani and K. N. Kudin, "Electronic response properties of carbon nanotubes in magnetic fields," ACS Nano, vol. 2, no. 4, pp. 661-668, 2008.

[33] O. V. Yazye and L. Helm, "Isotropic Knight shift of metallic carbon nanotubes," Physical Review B, vol. 72, no. 24, Article ID 245416, 5 pages, 2005.

[34] Y. Ihara, P. Wzietek, H. Alloul, M. H. Rummeli, Th. Pichler, and F. Simon, "Incidence of the Tomonaga-Luttinger liquid state on the NMR spin lattice relaxation in Carbon Nanotubes," Europhysics Letters, vol. 90, article 17004, 2010.

[35] B. Dóra, M. Gulácsi, F. Simon, and H. Kuzmany, "Spin gap and luttinger liquid description of the NMR relaxation in carbon nanotubes," Physical Review Letters, vol. 99, no. 16, Article ID 166402, 4 pages, 2007.

[36] E. Abou-Hamad, C. Goze-Bac, F. Nitze et al., "Electronic properties of Cs-intercalated single-walled carbon nanotubes derived from nuclear magnetic resonance," New Journal of Physics, vol. 13, Article ID 053045, 2011.

[37] M. Bouhrara, Y. Saih, T. Wågberg, C. Goze-Bac, and E. AbouHamad, "High-resolution ${ }^{13} \mathrm{C}$ nuclear magnetic resonance evidence of phase transition of Rb,Cs-intercalated single-walled nanotubes," Journal of Applied Physics, vol. 110, no. 5, Article ID 054306, 2011.

[38] X.-P. Tang, A. Kleinhammes, H. Shimoda et al., "Electronic structures of single-walled carbon nanotubes determined by NMR," Science, vol. 288, no. 5465, pp. 492-494, 2000.

[39] J. W. Mintmire and C. T. White, "First-principles band structures of armchair nanotubes," Applied Physics A, vol. 67, no. 1, pp. 65-69, 1998. 

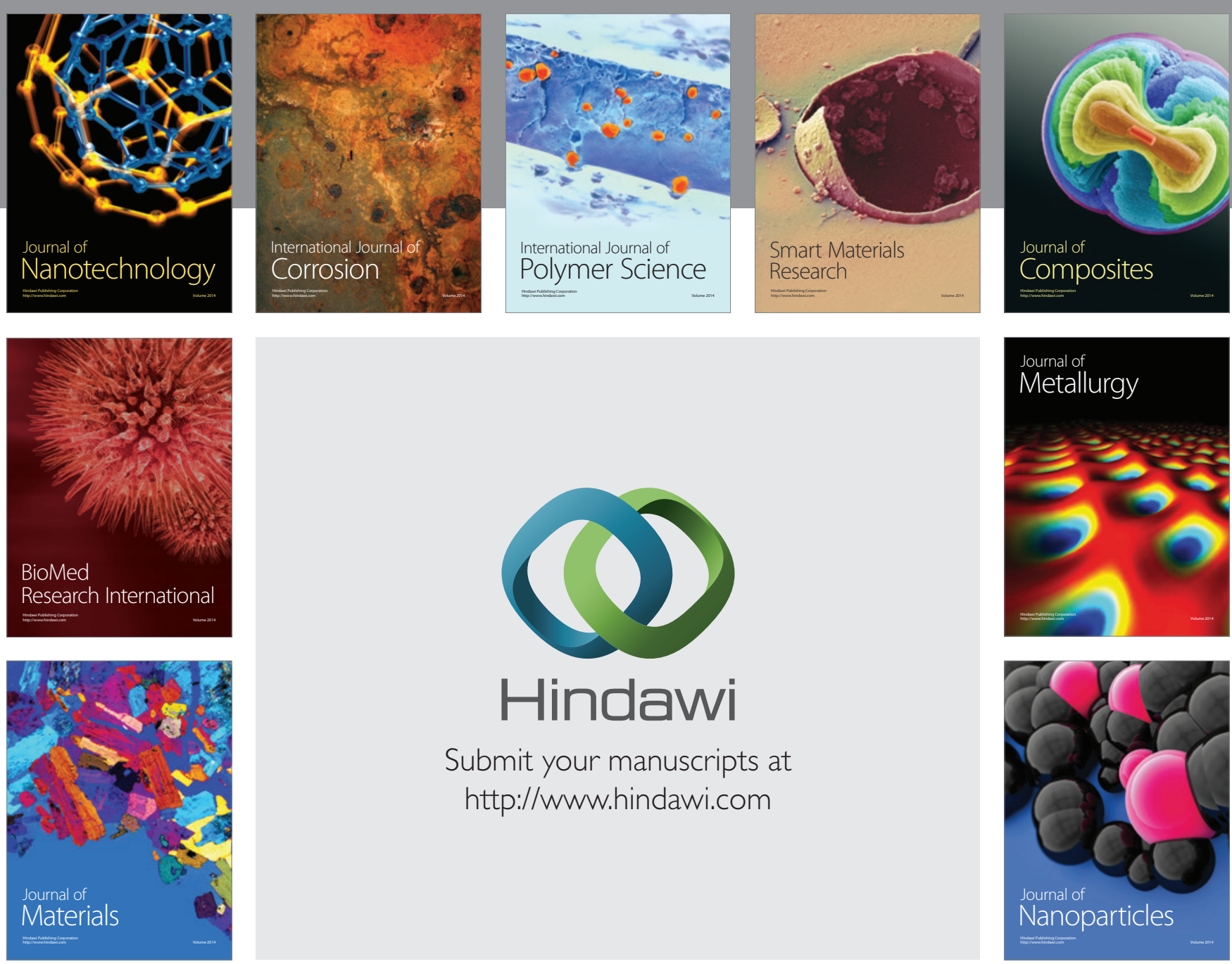

Submit your manuscripts at http://www.hindawi.com
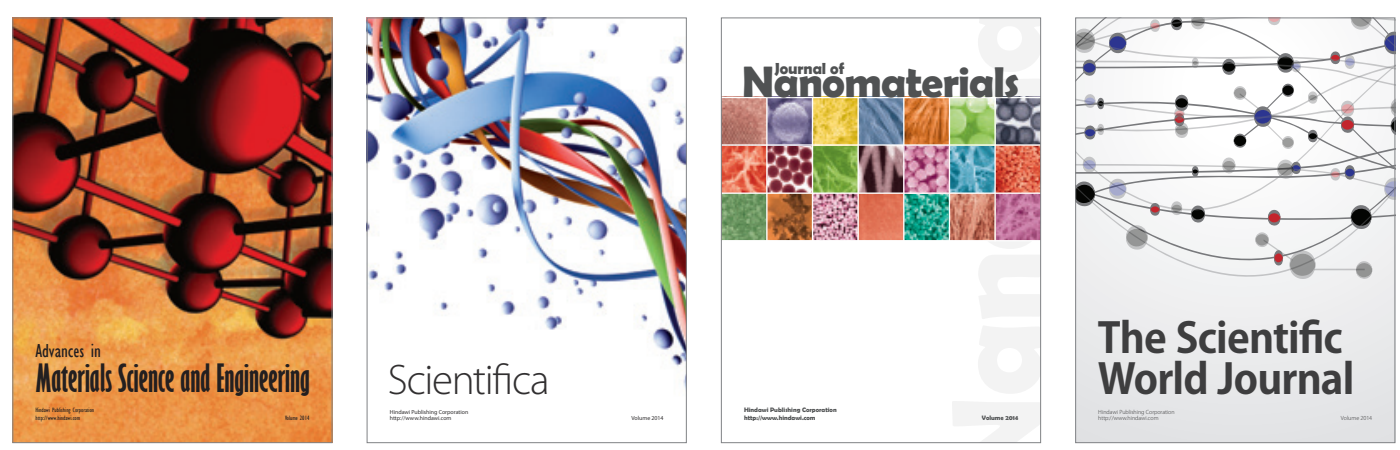

\section{The Scientific World Journal}
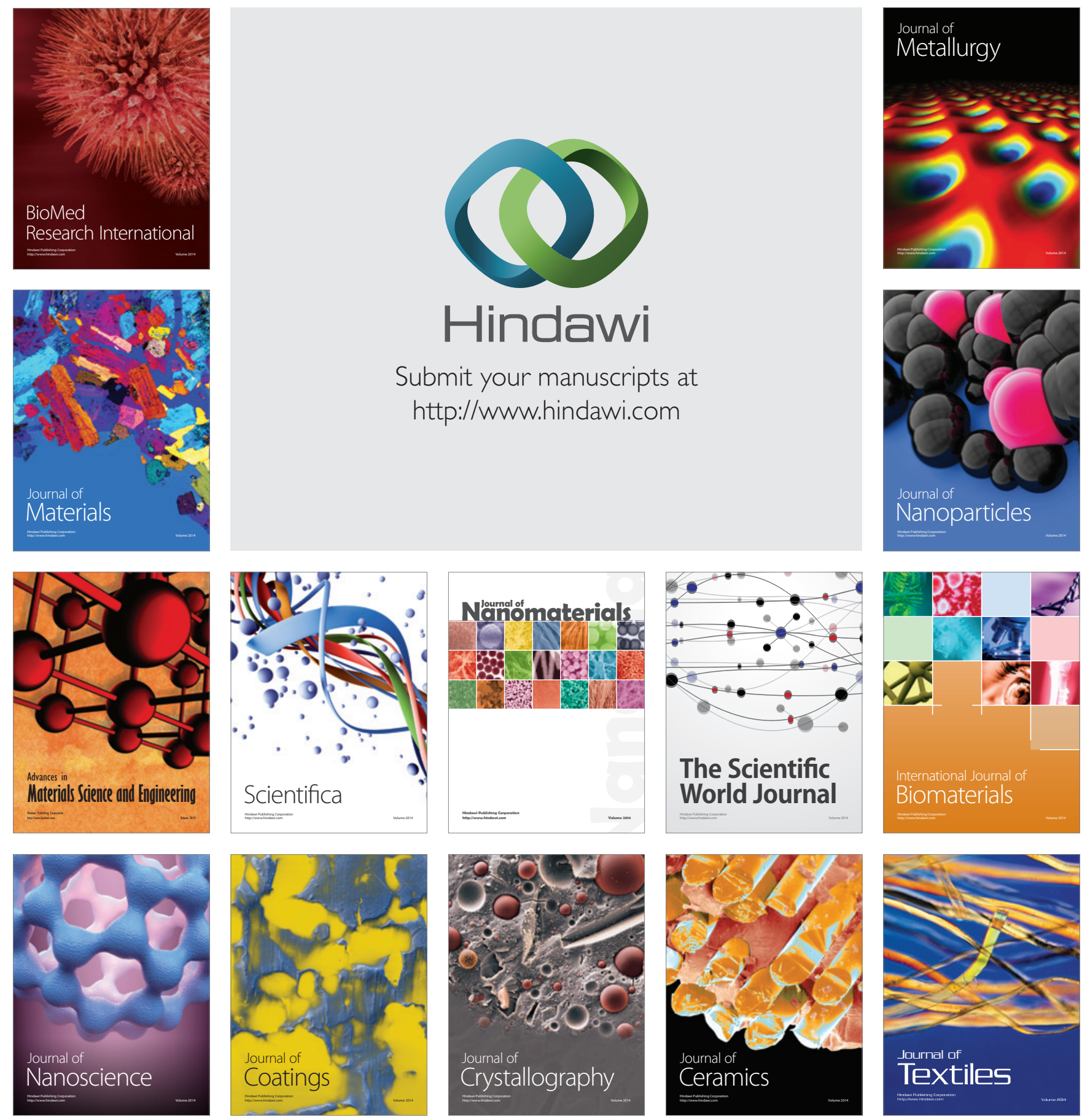